\title{
Determination of Blood Physiological and Biochemical Values of Blue Peafowl
}

\author{
Xu Xiao-xia ${ }^{1}$, Qian Guo-hong ${ }^{2}$, Yang Fu-min ${ }^{1, *}$, Yang Min ${ }^{3}$, Wang Xue-yan ${ }^{1}$ \\ ${ }^{1}$ College of Food Science and Engineering, Gansu Agricultural University, Lanzhou, China \\ ${ }^{2}$ Gansu Provincial Health Education Institute, Lanzhou, China \\ ${ }^{3}$ College of Science, Gansu Agricultural University, Lanzhou, China \\ *Corresponding author: yfumin@gsau.edu.cn
}

Received November 30, 2014; Revised January 15, 2015; Accepted February 04, 2015

\begin{abstract}
Forty-nine physiological and biochemical indices of blood in blue peafowl were determined in this report. Significant differences were observed in the hematocrit, alkaline phosphatase, CO2-M and anion gap ( $p<$ 0.01 ), as well as mean corpuscular hemoglobin, a-hydroxybutyrate dehydrogenase, $\mathrm{Mg}^{2+}$, erythrocyte number, glutamicpuruvic transaminase, total protein and total bile acid $(p<0.05)$ of different sexes. The results may provide a reference for feeding, health examination, diagnosis, and breeding of blue peafowl.
\end{abstract}

Keywords: blue peafowl, blood, physiological values, biochemical values

Cite This Article: Xu Xiao-xia, Qian Guo-hong, Yang Fu-min, Yang Min, and Wang Xue-yan, "Determination of Blood Physiological and Biochemical Values of Blue Peafowl." Journal of Food and Nutrition Research, vol. 3, no. 2 (2015): 104-108. doi: 10.12691/jfnr-3-2-5.

\section{Introduction}

The peafowl belongs to phasianidae, pavo, a kind of special poultry breed for viewing and admiring [1,2,3]. Peafowl meat has been verified possess detoxifying effect [3]. As recorded in a well-known Chinese medicine book "Outline Treatise of Medical Herbs", peafowl meat is a kind of medicinal diet with the functions of eliminating toxin, beautifying skin [4]. There are two kinds of peafowl in the world, blue peafowl(Pavo critatus) and green peafowl(Pavo muticus). Compared to green peafowl, the artificial domestication of blue peafowl is earlier than green peafowl, which has a history of 3000 years. Blue peafowl has a higher reproductive capacity under appropriate breeding and management. Wild green peafowl is listed as the national first-class protected animals in China, while home-raised blue peafowl is not included, so they can be raised for edible and medical use. Blue peafowl has breed in the zoo of most countries in recent years [5]. In China, blue peafowl breeding was paid much attention because it has extensive adaptability, higher disease resistance, crude feed tolerance and high economic benefits. Researches on blue peafowl has been listed as a promotion project of Star Program by the State Ministry of Science \&Technology, and also has been designed as special livestock and poultry industrial management program by China's State Forestry Administration [4].

The literature focusing on blue peafowl are mainly about breeding, disease diagnosis and treatment [6,7], nutrition [5,8-13], muscle histological characteristics [14], slaughter performance, meat quality traits [4], and genetic diversity [15-20]. However, the determination physiological and biochemical values of blue peafowl has not been reported. Physiological and biochemical values are important indexes in medical biology research, which can be used to evaluate animal health, and also applied as the important reference of pathology and toxicology studies $[21,22]$. The paper aimed to determine and compare the blood physiological and biochemical values between male and female blue peafowl. The results can provide reference and theoretical basis for artificial breeding, ecological protection, raising and management, epidemic disease prevention and cure of blue peafowl.

\section{Materials and Methods}

\subsection{Blue Peafowl}

The experiment was carried out by using twelve blue peafowl ( 6 males and 6 females, one-year old, mean body weight were $4.314 \mathrm{~kg}$ and $2.755 \mathrm{~kg}$, respectively) from Gansu Hongxiang blue peafowl high-tech agricultural development Co. Ltd in Gansu Province, China. Blue peafowl were subjected to clinical examination before study and only healthy subjects were used.

\subsection{Blood Sampling}

Blood samples $(5 \mathrm{~mL}$ ) were collected by wing venipuncture before morning feeding (each peafowl was sampled once) in two different vacutainer tubes. To assess blood physiological values, samples were collected in $1 \mathrm{ml}$ vacutainer test tubes containing $0.1 \%$ heparin anticoagulant. This allowed us to analyze the blood without the risk of clotting in the tube. The rest $4 \mathrm{ml}$ in vacutainer were 
centrifuged at 3000r for $15 \mathrm{~min}$, within $30 \mathrm{~min}$ for the collection and the obtained serum was stored at $-20^{\circ} \mathrm{C}$ until biochemical analyses.

\subsection{Determination of Blood Physiological and Biochemical Values}

\subsubsection{Blood Physiological Values}

Blood physiological values including Red blood cell count(RBC), White blood cell count(WBC), Hemoglobin (HB), Hematocrit (HCT), Mean corpuscular volume (MCV), Mean corpuscular hemoglobin(MCH), Hemoglobin concentration (MCHC), Red cell distribution width (RDW-CV), Platelet count(PLT), Lymphocyte ratio(LYM\%), Lymphocyte count (LYM) of blue peafowl blood were determined using an automatic blood cell analyzer (JN1212-ABX-MICROS 60, France).

\subsubsection{Blood Biochemical Values}

The serum was separated and used for the assay of 29 biochemical attributes including Creatine kinase(CK), Creatine kinase-MB(CK-MB), $\alpha$-hydroxybutyric dehydrogenase $(\alpha-\mathrm{HBDH})$, Alanine minotransferase (ALT), etc. with standard analytical procedures. The reagent kits supplied by Biological Engineering Co., Ltd. Beijing, China were utilized for the analysis of biochemical attributes with automatic biochemical analyzer (AMS-18, Italian). Electrolyte analyzer (HX-7185, Hengxing Technology Development Co., Ltd. Heifei, China) was used to analyze the content of $\mathrm{K}, \mathrm{Na}, \mathrm{Cl}, \mathrm{iCa}, \mathrm{Ca}, \mathrm{Mg}, \mathrm{P}, \mathrm{CO} 2-\mathrm{M}$ ) and AG of blue peafowl blood, and the operation can refer to specification supplied with the above mentioned instruments.

\subsection{Statistical Analysis}

All the measurements were carried out in triplicate. The values were averaged and reported along with their standard deviation (S.D). Data were analyzed with paired $t$ test. Probabilities lower than 0.01 were considered as statistically highly significant $(p<0.01)$. Probabilities lower than 0.05 were considered as statistically significant $(p<0.05)$. All statistical calculations were performed with the SPSS 13.0 statistical software for Windows.

\section{Results and Discussion}

\subsection{Blood Physiological Values of Blue Peafowl}

Blood physiological values of blue peafowl were shown in Table 1 . MCV was very significantly different $(p<0.01)$ between male and female blue peafowl, RBC and $\mathrm{MCH}$ were significantly different $(p<0.05)$ between male and female blue peafowl blood, while other physiological values were not significantly different $(p>0.05)$ between male and female.

The normal values of blood physiological are the main indicators that reflect body health, it is also one of the index for diagnosis and monitoring of diseases. The number and size of RBC as well as the content of $\mathrm{HB}$ reflect oxygen-transport ability of the animal [23]. The number of RBC is not fixed, and can change in response to factors such as breed, age, gender, physical status, living environment and other factors. In general, the number of RBC in young animals is higher than that of adult animals, the number of RBC in male animals is higher than that of female animals, the number of RBC in good nutrition animals is higher than that of malnutrition. Many studies have found that hungry animals contain less $\mathrm{RBC}$ and $\mathrm{HB}$ as well as low metabolism. This is the instinctive response of hungry animals to reduce the use of stored energy in order to maintain basic life activities [24].

Table 1. Blood biochemical indices of blue peafowl ( $\bar{X} \pm s, \mathbf{n}=3$ )

\begin{tabular}{lrrc}
\hline Items & Male blue peafowl & Female blue peafowl & $\boldsymbol{p}$-value \\
\hline WBC $\left(10^{9} / \mathrm{L}\right)$ & $219.467 \pm 8.262$ & $227.600 \pm 6.634$ & $N S$ \\
RBC $\left(10^{12} / \mathrm{L}\right)$ & $1.397 \pm 0.085$ & $1.593 \pm 0.049$ & $*$ \\
HGB $(\mathrm{g} / \mathrm{L})$ & $119.000 \pm 9.644$ & $116.333 \pm 6.027$ & $N S$ \\
HCT & $25.100 \pm 1.637$ & $26.867 \pm 0.568$ & $N S$ \\
MCV(fL) & $179.700 \pm 2.893$ & $168.700 \pm 2.291$ & $* *$ \\
MCH(pg) & $85.200 \pm 4.503$ & $73.133 \pm 5.871$ & $*$ \\
MCHC (g/L) & $473.667 \pm 17.926$ & $433.333 \pm 29.704$ & $N S$ \\
RDW-CV (\%) & $8.600 \pm 0.889$ & $10.733 \pm 1.209$ & $N S$ \\
PLT $\left(10^{9} / \mathrm{L}\right)$ & $28.333 \pm 1.527$ & $30.000 \pm 1.000$ & $N S$ \\
LYM\% $(\%)$ & $95.800 \pm 1.126$ & $62.367 \pm 4.039$ & $N S$ \\
LYM $\left(10^{9} / \mathrm{L}\right)$ & $210.300 \pm 9.417$ & $140.433 \pm 21.919$ & $N S$ \\
\hline
\end{tabular}

${ }^{* *} p<0.01 ; * p<0.05$; NS $p \geq 0.05$.

The results showed that the RBC of male was significantly lower than that of female $(p<0.05)$, the $\mathrm{MCH}$ of male was significantly higher than that of female $(p<0.05)$, the MCV of male was very significantly higher than that of female $(p<0.01)$. Frequent and intensive activities as well as big oxygen consumption of male blue peafowl have caused these results. WBC mainly take part in immune response for body, and it can resist the invasion of the bacteria, viruses and other pathogens. The results of WBC, LYM and LYM \% were not significantly different $(p>0.05)$ between male and female showed the immunity of male and female is basically equal.

\subsection{Blood Biochemical Values of Blue Peafowl}

\subsubsection{Blood Enzyme Activities}

Blood enzyme activities of blue peafowl were shown in Table 2. Except a-HBDH, ALT and ALP, other enzyme activities were not significantly different $(p>0.05)$ between male and female.

Serum enzyme is an important sign of the integrity of the cell membrane function. The changes of serum enzyme activity reflect the status of metabolism of material and the different states of organizational structure function [25,26]. Analyzing the activity and change rule of serum enzyme is of great practical significance to understand the animal growth and breeding character as well as meat quality improvement $[27,28]$. Studies have reported that ALT activities are positively associated with slaughter performance, breast meat rate, thigh meat rate, lean meat rate and belly fat percentage [29]. ALT has a very important function in amino metabolism as well as in protein, fat and glucose metabolism. The activities of ALT in serum are normally very low, but its levels in the blood 
would increase when the liver tissue is damaged or diseased. Thus, ALT can be used as an indicator for clinical diagnosis. ALP is an important enzyme widely distributed in the animal blood and organs, catalyzing various phosphates and release of inorganic phosphorus. ALP plays a central role in bone formation and fat synthesis, so it is an important index for the physiological activity and related disease diagnosis $[28,30]$. For example, the serum level of ALP is an index for evaluating the metabolism status of liver and kidney which become active as the content of ALP increasing. HBDH mainly comes from cardiac, kidney and red blood cells, among which cardiac tissue contains the most abundant, so the HBDH activity of blood was apparently elevated cardiac diseases. Additionally, $\mathrm{HBDH}$ is an isozyme of $\mathrm{LDH}$, which activity changes is paralleled with the LDH [31,32]. The serum level of HBDH is also an important index for evaluating the nutrition status.

Table 2. Enzyme activity in blood of blue peafowl ( $\bar{X} \pm s, \mathbf{n}=3$ )

\begin{tabular}{lrrc}
\hline Items & Male blue peafowl & Female blue peafowl & $\boldsymbol{p}$-value \\
\hline CK (U/L) & $1684.333 \pm 233.432$ & $1508.667 \pm 503.701$ & $N S$ \\
CK-MB (U/L) & $2982.333 \pm 415.587$ & $2659.333 \pm 882.413$ & $N S$ \\
HBDH (U/L) & $110.333 \pm 17.926$ & $69.000 \pm 15.524$ & $*$ \\
LDH (IU/L) & $196.000 \pm 57.035$ & $137.000 \pm 74.987$ & $N S$ \\
ALT (U/L) & $2.667 \pm 0.619$ & $9.333 \pm 3.650$ & $*$ \\
AST (U/L) & $106.667 \pm 7.234$ & $137.000 \pm 42.036$ & $N S$ \\
CHE (U/L) & $3522.333 \pm 598.640$ & $3317.333 \pm 765.569$ & $N S$ \\
ALP (U/L) & $140.333 \pm 97.961$ & $29.000 \pm 2.000$ & $* *$ \\
GGT (U/L) & $2.333 \pm 0.577$ & $2.333 \pm 1.309$ & $N S$ \\
AMY (U/L) & $635.666 \pm 40.251$ & $644.333 \pm 5.508$ & $N S$ \\
\hline
\end{tabular}

${ }^{* *} p<0.01 ; * p<0.05$; NS $p \geq 0.05$.

The results show that the ALP of male was very significantly higher than that of female $(p<0.01)$, but the ALT of male was significantly lower than that of female $(p<0.05)$. The HBDH of male was significantly higher than that of female $(p<0.05)$, which are not in accordance with Zhou Qin-ping's conclusion that no significant difference of ALT was between males and females ( $p>$ $0.05)$ [28]. The HBDH of male was significantly higher than that of female might be one reason for the difference between the sexes.

\subsubsection{Blood Organic Content}

Blood organic content of blue peafowl were shown in Table 3. Except TP and TBA, other organic content were not significantly different $(p>0.05)$ between male and female.

$\mathrm{TP}$ is an effective index to reflect the protein metabolism of poultry. High TP content of the serum reflects protein metabolism activity, which is conductive to improve the protein absorption and promote the animal's growth [33]. The TP content was influenced by dietary protein content to a certain extent, which related to energetic materials, and also has relationship with age, physiological state and health status [34,35]. The content of TP and album are reduced in malnutrition animals, while high-protein diets will raise the content of urea nitrogen [36,37].

The results showed that the TP of male was significantly lower than that of female $(p<0.05)$, it may be attributed to multi-factors including physiological metabolism, movement difference, and so on. The TBA is formed by cholesterol, which is the important basis for judging animal liver disease. The results showed that the TBA of male was significantly lower than that of female $(p<0.05)$. The difference of blood organic content between male and female may attribute to sexes and take feeding status. Therefore, we cannot conform diseases rely on sexes when use the changes of organic content as diagnostic indexes in practice. We need to increase the protein levels of feeds in the feeding.

Table 3. Blood organic content of blue peafowl ( $\bar{X} \pm s, \mathbf{n}=3$ )

\begin{tabular}{|c|c|c|c|}
\hline Items & Male blue peafowl & Female blue peafowl & $p$-value \\
\hline GLU (mmol/L) & $18.100 \pm 0.625$ & $17.500 \pm 1.418$ & NS \\
\hline GSP (mmol/L) & $1.467 \pm 0.116$ & $1.500 \pm 0.200$ & NS \\
\hline BUN (mmol/L) & $0.267 \pm 0.058$ & $0.433 \pm 0.153$ & NS \\
\hline CRE (umol/L) & $16.300 \pm 5.032$ & $22.233 \pm 10.504$ & NS \\
\hline BUN/CL & $4.780 \pm 2.352$ & $5.247 \pm 1.025$ & NS \\
\hline UA (umol) & $163.333 \pm 62.565$ & $213.667 \pm 71.598$ & NS \\
\hline $\mathrm{TCH}(\mathrm{mmol} / \mathrm{L})$ & $2.800 \pm 0.625$ & $4.067 \pm 1.343$ & NS \\
\hline $\mathrm{TG}(\mathrm{mmol} / \mathrm{L})$ & $1.633 \pm 0.116$ & $2.000 \pm 0.557$ & NS \\
\hline HDL (mmol/L) & $1.107 \pm 0.317$ & $1.457 \pm 0.144$ & NS \\
\hline LDL (mmol/L) & $1.377 \pm 0.231$ & $2.417 \pm 1.649$ & NS \\
\hline APO A1 (g/L) & $0.080 \pm 0.003$ & $0.093 \pm 0.015$ & NS \\
\hline APOB $(g / L)$ & $0.063 \pm 0.038$ & $0.053 \pm 0.040$ & NS \\
\hline APO-A( A1/B) & $1.963 \pm 0.765$ & $4.443 \pm 2.681$ & NS \\
\hline $\mathrm{TP}(\mathrm{g} / \mathrm{L})$ & $33.533 \pm 4.680$ & $49.300 \pm 7.436$ & * \\
\hline $\operatorname{ALB}(g / L)$ & $17.600 \pm 3.396$ & $21.367 \pm 0.503$ & NS \\
\hline TBIL (umol/L) & $3.400 \pm 0.400$ & $4.733 \pm 0.757$ & NS \\
\hline DBI (umol/L) & $1.900 \pm 0.600$ & $2.733 \pm 0.473$ & NS \\
\hline IBIL (umol/L) & $1.500 \pm 0.872$ & $2.000 \pm 0.819$ & NS \\
\hline TBA (umol/L) & $30.333 \pm 15.502$ & $57.333 \pm 5.132$ & $*$ \\
\hline
\end{tabular}

\subsubsection{Blood Electrolyte Content}

Water and electrolyte are main composition in body fluids, and electrolyte content is varied with different gender and age. Electrolytes are important to keep the balance of salts in animal body. The concentration of plasma electrolytes plays a key role maintaining cell metabolism, osmotic pressure, proper water distribution, body acid-base balance, neuromuscular excitement, blood $\mathrm{pH}$, muscular excitement and the status of heart and kidney [23,38].

Blood electrolyte content of blue peafowl are shown in Table 4. CO2-M and AG of male were very significantly higher than that of female $(p<0.01)$, the $\mathrm{Mg}$ of male was significantly higher than that of female $(p<0.05)$, while $K$, $\mathrm{Na}, \mathrm{Ca}$, and $\mathrm{P}$ content were not significantly different $(p>$ 0.05 ) between male and female. Some researchers reported that inorganic phosphorous content in serum of Crossoptilon mantchuricum was significantly different ( $p$ $<0.05$ ) between male and female [39]. The $\mathrm{Cl}$ and $\mathrm{Na}$ content of male chrysolophus pictus were significantly higher than those of female $(p<0.05)$ [23]. The K content of male cabot's tragopan was higher than that of female (male $62.57 \mathrm{mg} / \mathrm{L}$, femal $153.41 \mathrm{mg} / \mathrm{L}$ ), while the Ca content of male cabot's tragopan was lower than that of 
female (male $136.21 \mathrm{mg} / \mathrm{L}$, femal $145.53 \mathrm{mg} / \mathrm{L}$ ) [40]. It shows that the concentration of plasma electrolytes may be different among animals, and it also suggests that body was in abnormality.

Table 4. Blood electrolyte content of blue peafowl $(\bar{x} \pm s, \mathbf{n}=3)$

\begin{tabular}{lrrr}
\hline Items & $\begin{array}{r}\text { Male blue } \\
\text { peafowl }\end{array}$ & $\begin{array}{r}\text { Female blue } \\
\text { peafowl }\end{array}$ & $\boldsymbol{p}$-value \\
\hline $\mathrm{K}(\mathrm{mmol} / \mathrm{L})$ & $3.183 \pm 0.032$ & $3.893 \pm 0.667$ & $N S$ \\
$\mathrm{Na}(\mathrm{mmol} / \mathrm{L})$ & $149.667 \pm 0.577$ & $150.000 \pm 2.000$ & $N S$ \\
$\mathrm{Cl}(\mathrm{mmol} / \mathrm{L})$ & $102.000 \pm 0.006$ & $102.667 \pm 1.528$ & $N S$ \\
$\mathrm{iCa}(\mathrm{mmol} / \mathrm{L})$ & $1.393 \pm 0.006$ & $1.417 \pm 0.051$ & $N S$ \\
$\mathrm{Ca}(\mathrm{mmol} / \mathrm{L})$ & $2.787 \pm 0.011$ & $2.833 \pm 0.103$ & $N S$ \\
$\mathrm{Mg}(\mathrm{mmol} / \mathrm{L})$ & $0.617 \pm 0.129^{\mathrm{a}}$ & $0.403 \pm 0.015^{\mathrm{b}}$ & $*$ \\
$\mathrm{P}(\mathrm{mmol} / \mathrm{L})$ & $1.370 \pm 0.241$ & $1.667 \pm 0.258$ & $N S$ \\
$\mathrm{CO}{ }_{2}-\mathrm{M}(\mathrm{mmol} / \mathrm{L})$ & $29.933 \pm 2.424^{\mathrm{A}}$ & $20.227 \pm 1.395^{\mathrm{B}}$ & $* *$ \\
$\mathrm{AG}(\mathrm{mmol} / \mathrm{L})$ & $17.737 \pm 3.004^{\mathrm{A}}$ & $27.107 \pm 1.465^{\mathrm{B}}$ & $* *$ \\
\hline${ }^{* *} p<0.01 ;{ }^{*} p<0.05 ; \mathrm{NS} p \geq 0.05$. & & \\
\hline
\end{tabular}

\section{Conclusion}

11 of 49 physiological and biochemical indexes were showed significantly differences between male and female. Among them, the MCV, ALP, AG and CO2-M of male was very significantly higher than that of female $(p<$ 0.01 ), the $\mathrm{MCH}, \mathrm{HBDH}$ and $\mathrm{Mg}$ of male were significantly higher than those of female $(p<0.05)$. The RBC, ALT, TP and TBA of male were significantly lower than those of female $(p<0.05)$, while other index were not significantly different $(p>0.05)$ between male and female. It showed that there were differences existing in part physiological and biochemical indexes between male and female in normal circumstances. Thus, we should not use uniform standard to evaluate the status of nutrition, health and diseases diagnose of male and female blue peafowl when use blood physiological and biochemical indexes were referenced in practice.

\section{Acknowledgments}

The research was supported by the National Spark Program of the State Ministry of Science \&Technology (2008GA860013) and Scientists and Technicians Service Enterprise Project of the State Ministry of Science \&Technology (2009GJG10020). We are very grateful to Wei Chang-chun and Han Xue-rong (The Second Hospital of JiaYuGuan) for their technical assistance.

\section{References}

[1] Zhen Zuo-xin, “China animal data: Aves Vol. Fourth, Order Galliformes”, Science Press, 1978.

[2] Xie Pu, Xu Bao-min, Lou Yi-zhou, "Yunnan Special poultry production”, Yunnan Science and Technology Press, 1997.

[3] Lu Guang-lin, Sun Shu-xia, "The key point for feeding and management of peacock”, China Poultry, 33(12). 50-51. 2011.

[4] Yan Xiao-juan, Yang Fu-min, Guo Jian-hua, "Studies on carcass characteristics and meat quality of blue peafowl", Journal of Gansu Agriculture University, 44(4). 46-49. 2009.

[5] Ma Ze-fang, "Diet metabolic rate of young blue peafowl l", Journal of Northeast Forestry University, 29(5). 107-109. 2001.
[6] Lin Zhuo-hui, Fang Xiao-wen, Huang Sheng-ping, "Raising management of peacock", Poultry Husbandry and Disease Control, (5). 38-39. 1994.

[7] Wang Zong-huan, "Raising development and technique questions of peafowl”, The Jindun Publishing House, 2000.

[8] Dong Na, Fang Xiao-wen, Huang Sheng-ping, Gao Xiang, Ye Hong-wei, Cao Bing-hai, "Effects of dietary energy levels on egg production and fertility rate of blue peafowl breeder", Journal of China Agricultural University, 8(3). 95-98. 2003.

[9] Zou Qi, "Metabolic energy of conventional diet for adult blue peafowl”, Journal of Northeast Forestry University, 30(1). 57-59. 2002.

[10] Zou Qi, Liu Wei-shi, Tian Xiu-hua, Liang Qin-e, Liu Hua-rong, "Determination of protein level and nutrient metabolic ratio of winter diet for adult blue peafowl”, Journal of Northeast Forestry University, 31(6). 43-45. 2003.

[11] Dong Na, Fang Xiao-wen, He Ning, Wo Xu-min, Cao Bin-hai, "Comparative study on digestibility of dietary fibre in blue peafowl, turkey and cock", Chinese Journal of Animal Science, 40(12). 23-25. 2004.

[12] Dong Na, Wu Dong-bin, Fang Xiao-wen, Cao Bin-hai, "Effects of dietary energy levels on growth performance, energy metabolism and nitrogen metabolism of blue peafowl”, Heilongjiang Animal Science And veterinary Medicine, 6. 89-91. 2006.

[13] Wang Kang-wen, Wu Ke-bang, "Effects of different levels of methionine and lysine on growth performance of blue peafowl breeder”, Guangdong Agricultural Sciences, 4. 114-119. 2011.

[14] Li Chen-xi, Yang Fu-min, "Analysis on histological traits of muscle for blue peafowl”, Journal of Gansu Agriculture University, 48(6). 135-139. 2013.

[15] Ouyang Yi-na, Yang Ze-yu, Li Da-1in, Huo Jin-long, Qian Kun, Miao Yong-wang, "Genetic divergence between pavo muticus and pavo cristatus by Cyt b gene”, Journal of Yunnan Agricultural University, 24(2). 220-224. 2009.

[16] Chang Hong, Ke Ya-yong, Su Ying-juan, Zhang Guo-ping, Zhu Shi-Ji, "A study of the wild and captive green peafowl(Pavo muticus) by random- amplified polymorphic DNA", Hereditas, 24(3). 271-274. 2002.

[17] Chen Tao, Miao Yong-wang, Huo Jin-long, Wei Hong-jiang, Ye Lang-hui, Pan Wei-rong, Tian Ying-hua, "Genetic diversity of blue peafowl based on microsatellite markers", Journal of Yunnan Agricultural University, 21(3). 346-350. 2006.

[18] Bao Wen-bin, Chen Guo-hong, Shu Jing-ting, Xu Qi, Li Hui-fang, " Screening of peafowl microsatellite primers and analysis of genetic diversity”, Hereditas, 28(10). 1242-1246. 2006.

[19] Zhu Shi-jie, Chang-Hong, Zhang Guo-ping, Wang Wei, Xu Qifang, " Phylogenetic relationships among Pavo based on complete mitochondrial cytochrome b gene sequence variations", Acta Scientiarum Naturalium Universitatis Sunyatseni, 43(6). 45-47. 2004.

[20] Zou Fang-dong, Tong Xin-xin, Yue Bi-song, "Molecular cloning of activin $\beta A$ subunit mature peptide from peafowl and its application in taxonomy and phylogeny”, Hereditas, 27(2). 231235. 2005.

[21] Qi Yun, Cai Run-lan, Song Yang, "Effects of storage duration of blood sampling on serum biochemical measurement”, Chinese Journal of Comparative Medicine, 16(2). 73-77. 2006.

[22] Zeng Dan, Qiao Hong-guang, Ning Zhong-hua, “ Determination of values of physiological and biochemical parameters in dwarf brown Layer", China Animal Husbandry \& Veterinary Medicine, 37(9). 25-28. 2010.

[23] Chen Yu-qin, Yu Shi-yuan, "Some biochemical and physiological indices in blood of chrysolophus pictus, alectoris chukar and phasianus colchicus”, Acta Zoologica Sinica, 53(4). 674-681. 2007.

[24] Qian Yun-xia, Chen Hui-qun, Sun Jiang-fei, "Effects of starvation on hematological and blood biochemical indices in cultured lateolabrax japonicas”, Journal of Fishery Sciences of China, 9(2). 133-137. 2002.

[25] Ji De-wei, Li Ming-yun, Wang Tian-zhu, Zhang Cheng-nian, Xu Zhen, Xu Wan-tu, “ Effects of low temperature stress periods on serum biochemical indexes in large yellow croaker pseudosciaena crocea”, Fisheries Science, 28(1). 1-4. 2009.

[26] Ou You-jun, Fan Chun-yan, Li Jia-er, Yu Na, Su Hui, “Acute hypoxia stress on blood biochemical indexes in selective group of Trachinotus ovatus “, Acta Oceanologica Sinica, 36(4). 126-131. 2014. 
[27] Wu Yi-xiong, "Summary on biochemical indexes and productive performances poultry blood”, Guizhou Animal Science and Veterinary Medicine, 26(3). 12-13. 2002.

[28] Zhou Qing-ping, Chen Hong, Zhou Xue-lin, Huang Qian, "Determination on blood biochemical indices of pavo muticus", Hubei Agricultural Sciences, 50(15). 3214-3130. 2011.

[29] Chen Yan-shui, Liu Ding-fa, "The application of blood alkaline phosphatase polymorphisms of livestock in breeding”, Contemporary Animal Husbandry, 1. 4-6. 1999.

[30] Wu Wei, "The relationship between alkaline phosphatase and Laying Performance ”, Chinese Journal of Animal Science, 27(1). 15-17. 1991.

[31] Shao Jian-feng, Liu Zhong-min, Zhong Yong-gen, "Expression of $\alpha-\mathrm{HBDH}$ and $\mathrm{LDH}$ and its clinicopathological significance in leukemia patients”, Clinical Medicine of China, 19(8). 721-722. 2003.

[32] Cao De-man, "Diagnostic value of $\alpha-\mathrm{HBDH}$ in patients with acute myocardil infarction”, Chinese Medical Journal, 8(16). 1118-1118. 2006.

[33] Xu Liang-mei, Mou Shao-yang, Wang Yang, Duan Xiao-xue, Gao Xiang-min, Zhang Wei-wei, “ Effect of zinc acetate on growth performance, meal quality and serum biochemical index of broilers", Journal of Northeast Agricultural University, 44(12). 32-38. 2013.
[34] Liu Hua-zhong, Shen Qiu-gu, "Comparison of growth performance and serum protein level between Taihe silky fowls and Nancheng dark fowls”, Jiangxi Journal of Animal Husbandry \& Veterinary Medicine,1. 8-8. 2000.

[35] Liu Kang, Huang Kai, Qin Xi, Wu Hong-yu, Cheng Yuan, Huang Qing, Huang Xiu-yun, “ Effects of dietary carbohydrate levels on immunity and serum biochemical indices under low temperature stress in Tilapia (Oreochromis niloticus)”, Fisheries Science, 33(2). 88-91. 2014.

[36] Zhang Yun-mei, Wu Deng-hu, Yang Xiao-li, "The analysis of blood chemistry in peafowls with infectious laryngotracheitis”, Chinese Journal of Pest Control, 19(12). 705-707. 2003.

[37] Qi Wei, Li Yan-hong, Zhang Yu, "Determination of 18 biochemical values in blood for macaque under domestication”, Supplement to the Journal of Sun Yatsen, 1. 38-39. 1997.

[38] Dong Shu-qing, "Discussion on the relationship between kidney damage and the detected-values and ration of serum creatinine and urea nitrogen”, Guide of China Medicine, 36(9). 136-137. 2011.

[39] Tang Chao-zhong, Wen Wei-ye, Wei Ze-zhen, Yang Ai-lin, "Study on the physiological and biochemical indices of browneared pheasant (Crossoptilon mantchuricum) in the zoo", Chinese Journal of Applied and Environmental Biology, 4(1). 85-87. 1998.

[40] Li Li, Zhu Kai-min, Duan Wen-wu, Jiang Wei-xing, Fu Tongsheng, "Determination of biochemical and physiological values in blood of cabot's tragopan”, Chinese Journal of Zoology, 38(6). 9496. 2003. 